OUTP-92-10P

September 1992

\title{
Neutralino Dark Matter in a Class of Unified Theories
}

\author{
S.A. Abel and S. Sarkar \\ Theoretical Physics, \\ University of Oxford, \\ Oxford OX1 3NP, U.K. \\ I.B. Whittingham \\ Department of Physics \\ James Cook University \\ Townsville, Australia 4811
}

\begin{abstract}
The cosmological significance of the neutralino sector is studied for a class of supersymmetric grand unified theories in which electroweak symmetry breaking is seeded by a gauge singlet. Extensive use is made of the renormalization group equations to significantly reduce the parameter space, by deriving analytic expressions for all the supersymmetry-breaking couplings in terms of the universal gaugino mass $m_{1 / 2}$, the universal scalar mass $m_{0}$ and the coupling $A$. The composition of the lightest supersymmetric partner is determined exactly below the $\mathrm{W}$ mass, no approximations are made for sfermion masses, and all particle exchanges are considered in calculating the annihilation cross-section; the relic abundance is then obtained by an analytic approximation. We find that in these models, stable neutralinos may make a significant contribution to the dark matter in the universe.
\end{abstract}

(to appear in Nuclear Physics B) 


\section{Introduction}

The neutralino $(\chi)$ in supersymmetric theories is a leading candidate for the constituent of the dark matter in the universe [1]. It has been demonstrated to be the lightest supersymmetric partner (LSP), and to have a significant cosmological relic density, in both the minimal supersymmetric standard model (MSSM) [2]-[10], and in an extended version [11] in which symmetry breaking is driven by a gauge singlet [12]-114. In contrast to other dark matter candidates such as the axion or gravitino, the neutralino has a relic density $\rho_{\chi}$ such that its contribution to the cosmological density parameter, $\Omega_{\chi} \equiv \rho_{\chi} / \rho_{\mathrm{c}}$, is naturally close to unity [1]. Here $\rho_{\mathrm{c}} \simeq 1.05 \times 10^{-5} \mathrm{~h}^{2} \mathrm{GeV} \mathrm{cm}{ }^{-3}$ is the critical density corresponding to a flat universe and $h \equiv H_{0} / 100 \mathrm{~km} \mathrm{~s}^{-1} \mathrm{Mpc}^{-1}$ is the (conventionally scaled) Hubble parameter, which is observationally constrained to lie in the range $0.4 \lesssim h \lesssim 1.0$ [15].

The actual value of $\Omega$ is rather uncertain. Luminous matter, e.g. in the disks of galaxies, contributes at most $\Omega \sim 0.01$ while studies of dark matter in galactic halos and in groups and clusters of galaxies imply $\Omega \sim 0.01-0.2$ [16]. Indirect estimates of $\Omega$ based on observations of peculiar (i.e. non-Hubble) motions on supercluster and larger scales suggest higher values in the range $\sim 0.2-1$ [17]. The recent observations by the COBE satellite of angular fluctuations in the microwave background radiation has given strong support to the hypothesis that the universe is dominated by dark matter with a density close to the critical value; interpretations of these data require that for cold dark matter, $\Omega \sim(0.2-0.5) h^{-1}[18]$. Additionally, the product $\Omega h^{2}(\propto \rho)$ can be conservatively bounded from above by requiring that the age of the universe exceed the observational lower limit of $\sim 10^{10} \mathrm{yr}$; this yields $\Omega h^{2} \lesssim 1$ for $h \gtrsim 0.4$, assuming a Friedmann-RobertsonWalker cosmology [15]. Further, if one assumes that the dark matter provides the critical density, then the age constraint restricts the Hubble parameter to be $h \lesssim 2 / 3$, hence $\Omega h^{2} \lesssim 4 / 9$. Taking all this into account, we adopt as the range of cosmological interest:

$$
0.1 \lesssim \Omega_{\chi} h^{2} \lesssim 0.5 .
$$

Note that the range $0.01 \lesssim \Omega_{\chi} h^{2} \lesssim 0.1$ would be relevant only to the dark matter in galactic halos. Obviously the constituent of the cosmological (cold) dark matter may also be the constituent of the halo dark matter (but not neccessarily vice versa).

In the standard picture of the evolution of the universe [15], it is assumed that expansion is adiabiatic following an early period of inflation. Massive particles 'freeze out' of chemical equilibrium with the thermal radiation-dominated plasma when their rate of annihilation falls behind the rate of expansion; hence their relic abundance is inversely proportional to the thermally-averaged annihilation cross-section $\left\langle\sigma_{\text {ann }} v\right\rangle$. In order to expedite the calculation of this quantity in supersymmetric theories, most authors resort to assumptions about the values of various parameters in order to reduce the multidimensional parameter space (e.g. 20-dim in the case of the MSSM !). One common assumption, motivated by grand unified theories (GUT), is that the gaugino masses are related by their renormalisation from a common value at an energy scale of $\mathcal{O}\left(10^{16}\right) \mathrm{GeV}$, viz. 


$$
m_{\mathrm{Y}}=\frac{5}{3}\left(\frac{g_{\mathrm{Y}}^{2}}{g_{2}^{2}}\right) m_{2} .
$$

One can go further and note that all the low energy couplings are then related by the renormalization group equations (RGEs) and a few model-dependent parameters. Use of this fact is however seldom made in the literature, because the RGEs are not soluble analytically, and also because the large value suggested by experiment for the mass of the top quark requires its Yukawa coupling to be quite close to the unitarity limit in the MSSM, thus prohibiting any acceptable approximations.

On the other hand, attention has recently been drawn [8, 9, 10] to the importance of using accurate expressions for the low energy couplings when determining the relic abundance. The model considered in these papers was the MSSM as derived from supergravityinspired GUTs, which has only 6 arbitrary parameters defined at the unification scale. These are the universal scalar $\left(m_{0}\right)$ and gaugino $\left(m_{1 / 2}\right)$ masses, the Higgs coupling $(\mu)$ and the hidden sector parameters $(A$ and $B)$ and the top quark mass $\left(m_{\mathrm{t}}\right)$. In principle, the remaining parameters such as the Higgs vacuum expectation values (VEVs)

are determined by the electroweak breaking constraints, although in practice matters are complicated by large radiative corrections to the effective potential from top and stop diagrams [19].

In this paper we shall carry out a similar analysis for the 'minimally' extended supersymmetric standard model. Running the RGEs analytically, we have obtained polynomial approximations for all the parameters at the weak scale in terms of their values at the GUT scale. Comparison with numerical evaluations show our solutions to be accurate to better than $5 \%$, even close to the unitarity limit. This reduces the dimensionality of the parameter space to $6: m_{1 / 2}, m_{0}, A$, the top quark Yukawa coupling $\lambda_{2_{\mathrm{t}}}$, and two Yukawa couplings involving the Higgs fields and gauge singlets, $\lambda_{7}$ and $\lambda_{8}$. Again the Higgs VEVs are determinable from the electroweak symmetry breaking requirements. In the following section we describe the model and introduce the dark matter candidate. Then we review the prescription for analytically obtaining the relic density to an accuracy better than $5 \%$ and, using our RGE approximations, calculate its value for various choices of the Higgs VEVs. We find that the neutralino has a relic abundance $\Omega_{\chi} \sim \mathcal{O}(1)$ over most of the experimentally allowed parameter space.

\section{The Minimally Extended Standard Model}

The model we shall consider is the MSSM extended by including a gauge singlet to give the necessary Higgs mixing and thus break $\mathrm{SU}(2)_{\mathrm{L}} \otimes \mathrm{U}(1)_{\mathrm{Y}}$ [20, 21]. The relevant superpotential is

$$
\begin{aligned}
\mathcal{W}= & \lambda_{1} \mathrm{D}_{\mathrm{R}}\left(\mathrm{D}_{\mathrm{L}}^{\dagger} \mathrm{h}^{0}-\mathrm{U}_{\mathrm{L}}^{\dagger} \mathrm{h}^{-}\right)+\lambda_{2} \mathrm{U}_{\mathrm{R}}\left(\mathrm{U}_{\mathrm{L}}^{\dagger} \overline{\mathrm{h}}^{0}-\mathrm{D}_{\mathrm{L}}^{\dagger} \overline{\mathrm{h}}^{+}\right) \\
& +\lambda_{3} \mathrm{E}_{\mathrm{R}}\left(\mathrm{E}_{\mathrm{L}}^{\dagger} \mathrm{h}^{0}-\nu_{\mathrm{L}}^{\dagger} \mathrm{h}^{-}\right)+\lambda_{7}\left(\mathrm{~h}^{0} \overline{\mathrm{h}}^{0}-\mathrm{h}^{-} \overline{\mathrm{h}}^{+}\right) \phi_{0}+\lambda_{8} \phi_{0}^{3} .
\end{aligned}
$$


The first three terms give masses to the (s)quarks and (s)leptons, such that $\lambda_{1}\langle\mathrm{~h}\rangle, \lambda_{2}\langle\overline{\mathrm{h}}\rangle$ and $\lambda_{3}\langle\mathrm{~h}\rangle$ are the mass matrices of the down quarks, up quarks, and electrons respectively (the generation indices have been supressed). The form of eq. (3) is clearly similar to the Standard Model superpotential, apart from the presence of the last two couplings involving the gauge singlet $\phi_{0}$, which replace the usual quadratic Higgs coupling, and ensure a satisfactory breaking of $\mathrm{SU}(2)_{\mathrm{L}} \otimes \mathrm{U}(1)_{\mathrm{Y}}$. This model is the minimal extension to the MSSM that contains only trilinear couplings, thus making it a natural candidate to emerge from level-one fermionic string theory. (It should be pointed out that in the 'flipped string' models 22] no gauge singlet appears in the low-energy effective potential, because such singlets may aquire large VEVs at a scale of $\mathcal{O}\left(10^{11}\right) \mathrm{GeV}$ corresponding to the condensation of scalars in the hidden $\mathrm{SU}(4) \otimes \mathrm{SO}(10)$ sector. We are therefore not addressing schemes of this form.)

The phenomenology of such models has been studied extensively in the literature [12]-14, [21]-27, but relatively little attention has been paid to the cosmological relic abundance of the neutralinos therein. Other proposed dark matter candidates are the 'flatino' superpartner of the massless scalar flat field ('flaton') after SU(5) $\otimes \mathrm{U}(1)$ symmetry breaking [26] and the integer-charged bound states ('cryptons') in some hidden sector [27]. The flatino/neutralino mass matrix is restricted by the amount of R-parity breaking which is induced. This is because models with broken R-parity (such as the minimal flipped SU(5) model considered in ref. [26]) have relic particles which are unstable. The decays of such particles into photons, charged particles or even neutrinos may violate the astrophysical bounds obtained by analysis of the diffuse $\gamma$-ray background and data from underground and cosmic ray detectors [28]. Careful analysis of the diagonalisation of the neutral fermion mass matrix shows [29] that the mixing of the neutralino with the neutrino is $\mathcal{O}\left(m_{\mathrm{W}} / m_{\mathrm{GUT}}\right)^{2}$, not the generic value of $\mathcal{O}\left(m_{\mathrm{W}} / m_{\mathrm{GUT}}\right)$ adopted earlier [26]. Assuming that flaton decay repopulates the neutralino density, we find that the neutralino lifetime is $\approx 10^{6}-10^{15} \mathrm{yr}$ and falls in the region forbidden by the astrophysical bounds [28]. We are therefore forced to reject the flatino as a dark matter candidate, at least in the form presented in ref. [26]. There are few phenomenological restrictions on cryptons at present since their nominal cosmological abundance would be far in excess of cosmological limits and must needs be substantially diluted by invoking entropy generation subsequent to freeze-out. Hence their relic density is quite uncertain 27. Further, such particles can also decay through non-renormalizable superpotential interactions and are thus constrained by the aforementioned astrophysical bounds if their relic density happens to be comparable to that of the dark matter [28].

Inspired by the most general supergravity models [30], we shall break supersymmetry softly at a scale $m_{\mathrm{X}}$ with the initial conditions of universal gaugino masses $m_{1 / 2}$, scalar masses $m_{0}$ and trilinear scalar couplings $A$. For the sake of expediency we specifically assume $m_{\mathrm{X}}=m_{\mathrm{GUT}}$. However, we argue that changing the particular details of the model at high energies has only minor consequences for our analysis, and that we are therefore addressing a large class of possible theories. Consider, for example, the case where $m_{\mathrm{X}}=10^{-2} m_{\mathrm{GUT}}$. Between $m_{\mathrm{X}}$ and $m_{\mathrm{GUT}}$, the gauginos and squarks receive contributions from the RGEs of $\sim 5 \%$ of their final values, so that the assumed degeneracy of the soft breaking parameters is approximately correct. Likewise, we expect the effect 
of the deconfinement of any hidden sector to be mitigated if it happens reasonably close to the GUT scale. In addition, we observe the following: any parameters which do not depend on the values of the strong coupling or top quark Yukawa coupling, converge to infra-red stable points at low energy and are therefore relatively independent of the (dimensionless) details of the model at high energies. On the other hand parameters such as $\lambda_{7}$ which $d o$ depend on the strong coupling or top quark Yukawa coupling, are dominated by their (diverging) values at low energies and are thus also expected to be relatively stable to changes in the details of the model at high energy.

We shall only consider interactions relevant for neutralino masses below that of the $\mathrm{W}$ boson. (Above the $\mathrm{W}$ mass, the annihilation cross-section receives important contributions from processes such as $\chi \chi \rightarrow \mathrm{W}^{+} \mathrm{W}^{-}$四). Since the infra-red fixed point of $\lambda_{8}$ is only $\sim 0.21$ [11], we expect our analysis to be valid for most of the parameter space where $\left\langle\phi_{0}\right\rangle$ and $m_{1 / 2}$ are not too large. When the neutralino mass exceeds $m_{\mathrm{W}}$, our results are only qualitative.

If we neglect the bottom quark mass, then the squark and slepton mass matrices which we need are already diagonal, and are as follows:

the up and charm squarks,

$$
\left(\begin{array}{c}
m_{\tilde{\mathrm{u}}_{\mathrm{L}}}^{2} \\
m_{\tilde{\mathrm{u}}_{\mathrm{R}}}^{2}
\end{array}\right)=\left(\begin{array}{c}
m_{\mathrm{Q}}^{2}-g_{\mathrm{Y}}^{2}\left(\bar{v}^{2}-v^{2}\right) / 12+g_{2}^{2}\left(\bar{v}^{2}-v^{2}\right) / 4 \\
m_{\mathrm{U}}^{2}+g_{\mathrm{Y}}^{2}\left(\bar{v}^{2}-v^{2}\right) / 3
\end{array}\right),
$$

the down, strange and bottom squarks,

$$
\left(\begin{array}{c}
m_{\tilde{d}_{\mathrm{L}}}^{2} \\
m_{\tilde{d}_{\mathrm{R}}}^{2}
\end{array}\right)=\left(\begin{array}{c}
m_{\mathrm{Q}}^{2}-g_{\mathrm{Y}}^{2}\left(\bar{v}^{2}-v^{2}\right) / 12-g_{2}^{2}\left(\bar{v}^{2}-v^{2}\right) / 4 \\
m_{\mathrm{D}}^{2}-g_{\mathrm{Y}}^{2}\left(\bar{v}^{2}-v^{2}\right) / 6
\end{array}\right),
$$

the left and right handed sleptons,

$$
\left(\begin{array}{c}
m_{\tilde{\mathrm{e}}_{\mathrm{L}}}^{2} \\
m_{\tilde{\mathrm{e}}_{\mathrm{R}}}^{2}
\end{array}\right)=\left(\begin{array}{c}
m_{\mathrm{L}}^{2}+g_{\mathrm{Y}}^{2}\left(\bar{v}^{2}-v^{2}\right) / 4-g_{2}^{2}\left(\bar{v}^{2}-v^{2}\right) / 4 \\
m_{\mathrm{E}}^{2}-g_{\mathrm{Y}}^{2}\left(\bar{v}^{2}-v^{2}\right) / 2
\end{array}\right),
$$

and, the sneutrino,

$$
m_{\tilde{\nu}_{\mathrm{L}}}^{2}=m_{\mathrm{L}}^{2}+g_{\mathrm{Y}}^{2}\left(\bar{v}^{2}-v^{2}\right) / 4+g_{2}^{2}\left(\bar{v}^{2}-v^{2}\right) / 4
$$

where $v, \bar{v}$ and $x$ are the VEVs of the scalar fields $\mathrm{h}^{0}, \overline{\mathrm{h}}^{0}$ and $\phi_{0}$, and the rest of the notation is as in ref. [30.

In addition we have three Higgs states, whose mass squared matrix is: 


$$
\left(\begin{array}{ccc}
g^{2} v^{2}-\left(A_{7} \lambda_{7}+3 \lambda_{7} \lambda_{8} x\right) \bar{v} x / v & \left(2 \lambda_{7}^{2}-g^{2}\right) v \bar{v}+\left(A_{7}+3 \lambda_{8} x\right) \lambda_{7} x & \left(A_{7}+6 \lambda_{8} x\right) \lambda_{7} \bar{v}+2 \lambda_{7}^{2} v x \\
\left(2 \lambda_{7}^{2}-g^{2}\right) v \bar{v}+\left(A_{7}+3 \lambda_{8} x\right) \lambda_{7} x & g^{2} \bar{v}^{2}-\left(A_{7} \lambda_{7}+3 \lambda_{7} \lambda_{8} x\right) v x / \bar{v} & \left(A_{7}+6 \lambda_{8} x\right) \lambda_{7} v+2 \lambda_{7}^{2} \bar{v} x \\
\left(A_{7}+6 \lambda_{8} x\right) \lambda_{7} \bar{v}+2 \lambda_{7}^{2} v x & \left(A_{7}+6 \lambda_{8} x\right) \lambda_{7} v+2 \lambda_{7}^{2} \bar{v} x & 3 A_{8} \lambda_{8} x+36 \lambda_{8}^{2} x^{2}-A_{7} \lambda_{7} v \bar{v} / x
\end{array}\right)
$$

where $g^{2}=\left(g_{\mathrm{Y}}^{2}+g_{2}^{2}\right) / 2$, and we define, for future use: $\tan \beta_{x} \equiv x / \bar{v}, \tan \beta \equiv \bar{v} / v$. Finally there are two physical states coming from the pseudoscalar mass squared matrix,

$$
-\left(\begin{array}{cc}
\left(A_{7}+3 \lambda_{8} x\right) \lambda_{7} x V^{2} / v \bar{v} & \left(A_{7} \lambda_{7}-6 \lambda_{7} \lambda_{8} x\right) V \\
\left(A_{7} \lambda_{7}-6 \lambda_{7} \lambda_{8} x\right) V & \left(A_{7}+12 \lambda_{8} x\right) \lambda_{7} v \bar{v} / x+9 A_{8} \lambda_{8} x
\end{array}\right),
$$

where $V^{2}=\left(v^{2}+\bar{v}^{2}\right)$. The $A_{7}$ and $A_{8}$ are the coefficients of the $\lambda_{7}$ and $\lambda_{8}$ trilinear scalar coupling terms. In order to be able to identify the Goldstone boson state in the above, we have substituted in the minimisation conditions [11], and find the massless eigenvector to be

$$
\tilde{G}=\sin \beta \overline{\mathrm{h}}-\cos \beta \mathrm{h}
$$

as expected from consideration of current conservation. This use of the minimisation condition has the added advantage that it enables one to avoid estimating the SUSY breaking, gauge singlet mass-squared term, which is the most sensitive to small changes in the Yukawa couplings. As stated earlier, the remaining low energy parameters may be approximated to better than 5\% using the RGEs as follows.

We begin at $m_{\mathrm{W}}$ and renormalize the Yukawa and gauge couplings to $m_{\mathrm{GUT}}$ where $g_{3}$ and $g_{2}$ are unified. We have used 2-loop RGEs for the gauge couplings, and 1-loop RGEs for all other parameters, as in ref. [23]. At each step in the renormalization procedure, we keep track of the parameters by expanding them in terms of the Yukawa couplings at $m_{\mathrm{W}}$. Then the supersymmetry breaking terms $m_{1 / 2}, A$ and $m_{0}$ are switched on, and all the parameters evolved back down to $m_{\mathrm{W}}$. This gives a set of polynomials in $\lambda_{1}, \lambda_{2}, \lambda_{7}$, $\lambda_{8}, m_{1 / 2}, m_{0}$ and $A$ which we truncate at sixth order in the Yukawa couplings.

The current LEP values of the strong coupling (extracted from $\mathrm{Z}$ event shapes using resummed QCD) [32] and of the weak mixing angle [31] are given by :

$$
\alpha_{\mathrm{S}}\left(m_{\mathrm{Z}}\right)=0.124 \pm 0.005, \quad \sin ^{2} \theta_{\mathrm{W}}\left(m_{\mathrm{Z}}\right)=0.2325 \pm 0.0007,
$$

where both quantities are evaluated in the $\overline{\mathrm{MS}}$ scheme, as is appropriate for our renormalization group calculations. However, analyses of deep inelastic $\mu, \nu$ scattering give a somewhat smaller value: $\alpha_{\mathrm{s}}\left(m_{\mathrm{Z}}\right)=0.112 \pm 0.005$, while analyses of $\tau$ decays and of $\mathrm{J} / \Psi, \Upsilon$ decays give intermediate values, all with comparable errors [32]. Given the present uncertainty in determinations of $\alpha_{\mathrm{s}}$, we prefer to perform our calculations using the "weighted mean' value 32 


$$
\alpha_{\mathrm{s}}\left(m_{\mathrm{Z}}\right)=0.118
$$

with $\sin ^{2} \theta_{\mathrm{W}}\left(m_{\mathrm{Z}}\right)=0.2325$, and present the corresponding results in Appendix A. (Note that the equivalent expressions for the MSSM may be deduced by setting $\lambda_{7}=\lambda_{8}=0$, replacing $A_{7}$ by $B_{\mu}$, and the first term in $B_{\mu}$ by $B m_{0}$, where $B$ is the quadratic scalar Higgs coupling at the GUT scale.) In order to illustrate the effect of a small change in $\alpha_{\mathrm{s}}$, we also solve the RGEs using $\alpha_{\mathrm{s}}=1 / 8=0.125$ in Appendix B and using $\alpha_{\mathrm{s}}=0.113$ in Appendix C. It should be noted that since $\sqrt{\frac{5}{3}} g_{\mathrm{Y}}$ is still significantly below $g_{2}$ at the GUT scale, the value of $m_{\mathrm{Y}}$ is slight grey larger than suggested by eq. (2). In fact the correct relation is:

$$
m_{\mathrm{Y}}=\left(\frac{g_{2}^{2}(0)}{g_{\mathrm{Y}}^{2}(0)}\right)\left(\frac{g_{\mathrm{Y}}^{2}}{g_{2}^{2}}\right) m_{2} .
$$

These approximations turn out to be much better than expected, again because of the convergence of the couplings to infra-red stable points. Thus, despite the fact that the analytic approximations diverge from the correct values during the running of the RGEs towards the GUT scale, upon returning to the weak scale we find an accuracy of better than $5 \%$ when compared with numerical runnings, even close to the unitarity limit. The results obtained are sensitive to the value of $\alpha_{\mathrm{s}}$ and the current uncertainty $( \pm 0.005)$ in its determination corresponds to an uncertainty of $\sim 10 \%$ in the calculated couplings. We find a discrepancy of $\sim 10 \%$ with the results obtained using one-loop expressions for the gauge couplings (which for the first two generations reproduce the numerical values

obtained in ref. [9]). For the case $\alpha_{\mathrm{s}}=1 / 8$, we find good agreement with the numerical results of ref. [33], taking $\lambda_{2 \mathrm{t}}=0.31$.

\section{$3 \quad$ Relic Abundance of the Neutralino}

The relic neutralino energy density may be computed by solution of the Boltzmann transport equation governing the abundance of any stable particle in the expanding universe. The exercise is greatly simplified by noting that under suitable assumptions this reduces to the continuity equation 34

$$
\frac{\mathrm{d}}{\mathrm{d} t}\left(n_{\chi} R^{3}\right)=-\left\langle\sigma_{\mathrm{ann}} v\right\rangle\left[n_{\chi}^{2}-\left(n_{\chi}^{\mathrm{eq}}\right)^{2}\right] R^{3}
$$

this simply states that the number of neutralinos (of density $n_{\chi}$ ) in a comoving volume is governed by the competition between annihilations and creations, the latter being dependent, by detailed balance, on the equilibrium neutralino density, $n_{\chi}^{\mathrm{eq}}$. This equation holds if the annihilating particles are non-relativistic and if both the annihilating particles and the annihilation products are maintained in kinetic equilibrium with the background thermal plasma through rapid scattering processes [35, 36. The essential physical input is the 'thermally-averaged' annihilation cross-section, which may be conveniently calculated in the rest frame of one of the annihilating particles (i.e. the 'lab' frame) [36]: 


$$
\begin{aligned}
\left\langle\sigma_{\text {ann }} v\right\rangle & =\int_{0}^{\infty} \mathrm{d} \epsilon \mathcal{K}(x, \epsilon) \sigma_{\text {ann }} v, \\
\mathcal{K}(x, \epsilon) & \equiv \frac{2 x}{K_{2}^{2}(x)} \sqrt{\epsilon}(1+2 \epsilon) K_{1}(2 x \sqrt{1+\epsilon}), \quad x \equiv m_{\chi} / T, \quad \epsilon=\frac{s-4 m_{\chi}^{2}}{4 m_{\chi}^{2}},
\end{aligned}
$$

where $v$ is the relative velocity of the annihilating neutralinos, $s$ is the usual Mandelstam variable, and the $K_{n}$ are modified Bessel functions of order $n$. This 1-dim integral can be easily evaluated numerically and is well-behaved even if $\sigma_{\text {ann }} v$ varies rapidly with $\epsilon$, as near a resonance or at the opening up of a new annihilation channel. (Useful analytic approximations have been provided for these situations in refs. [36, 37].) Outside these problematic regions, a simple series expansion for $\left\langle\sigma_{\text {ann }} v\right\rangle$ is useful [36, 38]:

$$
\begin{aligned}
\left\langle\sigma_{\mathrm{ann}} v\right\rangle= & a^{(0)}+\frac{3}{2} a^{(1)} x^{-1}+\left(\frac{9}{2} a^{(1)}+\frac{15}{8} a^{(2)}\right) x^{-2} \\
& +\left(\frac{15}{16} a^{(1)}+\frac{195}{16} a^{(2)}+\frac{35}{16} a^{(3)}\right) x^{-3}+\cdots
\end{aligned}
$$

where $a^{(n)}$ is the $n$th derivative of $\left\langle\sigma_{\text {ann }} v\right\rangle$ with respect to $\epsilon$, evaluated at $\epsilon=0$. We will be interested in the value of $\left\langle\sigma_{\mathrm{ann}} v\right\rangle$ for very non-relativistic annihilating particles (with $x \sim 20$ ), hence it is adequate to retain terms only upto $\mathcal{O}\left(x^{-1}\right)$ if errors of a few percent are tolerable. Therefore we write

$$
\left\langle\sigma_{\mathrm{ann}} v\right\rangle \simeq a^{(0)}+\frac{3}{2} a^{(1)} x^{-1}
$$

Since the neutralino $(\chi)$ is a Majorana fermion, $a^{(0)}$ (the S-wave part in the above) is suppressed relative to $a^{(1)}$ (which contains both $\mathrm{S}$ and $\mathrm{P}$-wave pieces) due to the smallness of the final state fermion masses [2]. As noted above, this expansion breaks down close to resonances where the value of $a^{(1)}$ may even be negative; however careful analysis [36, 37] shows that the true situation is not in fact pathological. We are mainly interested in identifying the broad characteristics of parameter space, eq. (18) is therefore adequate for our purposes. The above expansion also breaks down close to particle thresholds because one of the denominators in $a^{(1)}$ decreases to zero. In our case this happens when the $\chi$ mass is close to that of the bottom quark; however as we will see, such low masses for $m_{\chi}$ are in fact disallowed by experiment. Finally, we stress again that only annihilation channels relevant for $m_{\chi} \lesssim m_{\mathrm{W}}$ are considered here. Above the $\mathrm{W}$ mass, $\left\langle\sigma_{\text {ann }} v\right\rangle$ receives important contributions from processes such as $\chi \chi \rightarrow \mathrm{W}^{+} \mathrm{W}^{-}$, as discussed in ref. [4]. This is relevant to the present discussion only for small values of $\tan \beta_{x}$ and large values of $m_{1 / 2}$, where our results are only qualitative.

Most solutions to the continuity equation (15) in the literature (e.g. [15, 34, 35]) have been obtained by transforming variables from $t \rightarrow T$ assuming that the product of the cosmic scale-factor $R$ and the photon temperature $T$ is constant. However, $R T$ does change as the temperature in the cooling universe falls below various mass thresholds and 
the corresponding particles annihilate and release entropy (i.e. thermalized interacting particles) into the system, according to

$$
\frac{\mathrm{d} R}{R}=-\frac{\mathrm{d} T}{T}-\frac{1}{3} \frac{\mathrm{d} g_{s_{\mathrm{I}}}}{g_{s_{\mathrm{I}}}}
$$

Here $g_{s_{\mathrm{I}}}$ counts the number of interacting degrees of freedom which determine the specific entropy density, $s_{\mathrm{I}}$ :

$$
\begin{aligned}
g_{s_{\mathrm{I}}}(T) & \equiv \frac{45}{2 \pi^{2}} \frac{s_{\mathrm{I}}(T)}{T^{3}}, \\
\text { where, } \quad s_{\mathrm{I}} & =\sum_{\text {int }} g_{i} \int \frac{3 m_{i}^{2}+4 p^{2}}{3 E_{i}(p) T} f_{i}^{\mathrm{eq}}(p, T) \frac{\mathrm{d}^{3} p}{(2 \pi)^{3}} .
\end{aligned}
$$

Here the sum is over all interacting particles, $E_{i} \equiv \sqrt{m_{i}^{2}+p^{2}}, g_{i}$ is the number of internal (spin) degrees of freedom, and the equilibrium phase-space distribution function (for zero chemical potential) is, $f_{i}^{\text {eq }}(p, T)=\left[\exp \left(E_{i} / T\right) \pm 1\right]^{-1}$, with $+/-$ corresponding to Fermi/Bose statistics.

In addition we have to keep track of any particle species which 'decouples', i.e. goes out of kinetic equilibrium with the thermal plasma, while still relativistic, thus taking its entropy content out of the 'interacting' sector (which defines the photon temperature). The quantity which remains truly invariant (in a comoving volume) is the total entropy $S=s R^{3}$, where

$$
s(T) \equiv \frac{2 \pi^{2}}{45} g_{s}(T) T^{3},
$$

with $g_{s}$, the entropy degrees of freedom, related to $g_{s_{\mathrm{I}}}$ as [36]

$$
g_{s}(T)=g_{s_{\mathrm{I}}}(T) \prod_{\text {dec }}\left[1+\frac{g_{s_{j}}\left(T_{\mathrm{D}_{j}}\right)}{g_{s_{\mathrm{I}}}\left(T_{\mathrm{D}_{j}}\right)}\right],
$$

The product is over all the decoupled particle species $j$ (which decouple at $T=T_{\mathrm{D}_{j}}$ ). Such particles will not share in any subsequent entropy release hence their temperature will drop below that of the interacting particles. By conservation of the total entropy, we obtain [36, 38] :

$$
\frac{T_{j}}{T}=\left[\frac{g_{s_{j}}\left(T_{\mathrm{D}}\right)}{g_{s_{j}}(T)} \frac{g_{s_{\mathrm{I}}}(T)}{g_{s_{\mathrm{I}}}\left(T_{\mathrm{D}}\right)}\right]^{1 / 3} .
$$

The total energy density includes, of course, all particles, decoupled or interacting, appropriately weighted by their respective temperatures. The energy degrees of freedom, $g_{\rho}$, is given by

$$
g_{\rho}(T) \equiv \frac{30}{\pi^{2}} \frac{\rho(T)}{T^{4}}
$$




$$
\text { where, } \quad \rho=\sum_{\text {all }} g_{k} \int E_{k}(q) f_{k}^{\mathrm{eq}}\left(p, T_{k}\right) \frac{\mathrm{d}^{3} p}{(2 \pi)^{3}} \text {. }
$$

In the present case the only particles which decouple while relativistic are massless neutrinos, hence $g_{s}$ is actually the same as $g_{s_{\mathrm{I}}}$ above the neutrino decoupling temperature of $\mathcal{O}(\mathrm{MeV})$, and can replace the latter in eq. (19) (see e.g. ref. [38])

The continuity equation (15) can now be rewritten [36] in terms of the dimensionless variables $Y_{\chi} \equiv n_{\chi} / s, Y_{\chi}^{\mathrm{eq}} \equiv n_{\chi}^{\mathrm{eq}} / s$, and $x \equiv m_{\chi} / T$ as

$$
\begin{aligned}
\frac{\mathrm{d} Y_{\chi}}{\mathrm{d} x} & =\lambda x^{-2}\left[\left(Y_{\chi}^{\mathrm{eq}}\right)^{2}-Y_{\chi}^{2}\right], \\
\text { where, } \quad \lambda & \equiv \sqrt{\frac{\pi}{45}} m_{\chi} m_{\mathrm{P}}\left\langle\sigma_{\mathrm{ann}} v\right\rangle g_{\star}^{1 / 2}, \\
\text { and, } \quad g_{\star}^{1 / 2}(T) & \equiv \frac{g_{s}(T)}{\sqrt{g_{\rho}(T)}}\left(1+\frac{1}{3} \frac{\mathrm{d} \ln g_{s_{\mathrm{I}}}(T)}{\mathrm{d} \ln T}\right) .
\end{aligned}
$$

The parameter $g_{\star}$ keeps track of the changing values of both $g_{\rho}$ (which measures the total energy density and thus determines the expansion rate $\dot{R} / R$ ), as well as $g_{s_{\mathrm{I}}}$ (which determines the specific entropy density and consequently the value of the adiabat $R T$ ). I The values of $g_{\rho}(T)$ and $g_{s_{\mathrm{I}}}(T)$ have been computed in ref. 38 by explicit integration over the particle distribution functions (using eqs. 20 and 24), and $g_{\star}(T)$ has been calculated from these data in ref. [36]. There is considerable ambiguity concerning the behaviour of these quantities during the quark-hadron phase transition and the authors of ref. [38] present curves for two choices of the critical temperature, $T_{\mathrm{c}}$, viz. $150 \mathrm{MeV}$ and $400 \mathrm{MeV}$ (corresponding to two choices of the 'bag' constant characterizing the confined hadrons). In fact the two curves merge at $T \gtrsim 800 \mathrm{MeV}$, above which temperature it is appropriate to treat the thermal plasma as an ideal gas of non-interacting fermions and gauge bosons. As we shall see, the cosmologically interesting regions of parameter space correspond to neutralino masses which are high enough $\left(m_{\chi} \gtrsim 20 \mathrm{GeV}\right)$ that freeze-out occurs well above the quark-hadron phase transition; hence their relic abundance is not particularly sensitive to the choice of $T_{c}$. To simplify the presentation of our results, we specifically adopt the curves corresponding to $T_{\mathrm{c}}=150 \mathrm{MeV}$. Another source of ambiguity concerns the electroweak phase transition, since if the relevant critical temperature is small then this may alter the relic abundance of massive particles which obtain their mass at this phase transition [39]. The LEP bounds on the Higgs mass imply that this does not happen in the minimal Standard Model [39], however in supersymmetric models, the electroweak phase transition may well be strongly supercooled. Nonetheless this ought not to affect the relic abundance of neutralinos lighter than a few hundred $\mathrm{GeV}$, which is of interest in this work. (However, such effects ought to be taken into account for the heavier neutralinos, $m_{\chi} \sim \mathcal{O}(\mathrm{TeV})$, considered in refs. [3, 4, 14].)

According to the continuity equation (25), the particle abundance tracks its equilibrium value as long as the self-annihilation rate is sufficiently rapid relative to the expansion

\footnotetext{
${ }^{1}$ Warning: $g_{\star}$ is often used (e.g. [15) to denote the energy degrees of freedom, which is called $g_{\rho}$ here.
} 
rate. When the particle becomes non-relativistic, its equilibrium abundance falls exponentially due to a Boltzmann factor, hence so does the annihilation rate. Eventually, the annihilation rate becomes sufficiently small that the particle abundance can no longer track its equilibrium value but becomes constant (in a comoving volume). Hence the parameter

$$
\Delta \equiv \frac{\left(Y_{\chi}-Y_{\chi}^{\mathrm{eq}}\right)}{Y_{\chi}^{\mathrm{eq}}},
$$

grows exponentially from zero as the particle goes out of chemical equilibrium, according to 36$]$

$$
\frac{45 g}{4 \pi^{4}} \frac{K_{2}(x)}{g_{s}} \lambda \Delta(\Delta+2)=\frac{K_{2}(x)}{K_{1}(x)}-\frac{1}{x} \frac{\mathrm{d} \ln g_{s_{\mathrm{I}}}}{\mathrm{d} \ln T},
$$

where we have used $Y_{\chi}^{\mathrm{eq}}=\left(45 \mathrm{~g} / 4 \pi^{4}\right)\left[x^{2} K_{2}(x) / g_{s}(T)\right]$. This equation can be solved for the 'freeze-out' temperature, $x=x_{\mathrm{fr}}$ corresponding to a specific choice of $\Delta=\Delta_{\mathrm{fr}}$; it is found that the choice $\Delta_{\mathrm{fr}}=1.5$ gives a good match to numerical solutions [36]. In fact, the first term on the r.h.s. is $\sim 1$ for non-relativistic particles, while the second term on the r.h.s. is zero and $g_{\star}^{1 / 2}=g_{\rho}^{-1 / 2}$ if $g_{s_{\mathrm{I}}}$ is constant at the epoch of freeze-out; the freeze-out temperature is then given by the more commonly used condition (e.g. 15]) :

$$
\begin{aligned}
x_{\mathrm{fr}} & =\ln \left[\Delta_{\mathrm{fr}}\left(2+\Delta_{\mathrm{fr}}\right) \delta\right]-\frac{1}{2} \ln x_{\mathrm{fr}} \\
\text { where } \delta & =\left(\frac{45}{32 \pi^{6}}\right)^{1 / 2} g m_{\chi} m_{\mathrm{P}}\left\langle\sigma_{\mathrm{ann}} v\right\rangle g_{\rho}^{-1 / 2} .
\end{aligned}
$$

This corresponds to the epoch when the annihilation rate, $n_{\chi}^{\mathrm{eq}}\left\langle\sigma_{\mathrm{ann}} v\right\rangle$, equals the rate of change of the particle abundance itself [34]: $\mathrm{d} \ln n^{\mathrm{eq}} / \mathrm{d} t=x_{\mathrm{fr}} \dot{R} / R$. We find that for neutralinos, $x_{\mathrm{fr}} \sim 20-25$.

After freeze-out, only annihilations are important since the temperature is now too low for the inverse creations to proceed; hence the asymptotic abundance, $Y_{\chi_{\infty}} \equiv Y_{\chi}(t \rightarrow \infty)$, obtains by integrating $\mathrm{d} Y_{\chi} / \mathrm{d} x=-\lambda x^{-2} Y_{\chi}^{2}$ with the initial condition, $Y_{\chi}\left(x_{\mathrm{fr}}\right)=Y_{\chi}^{\mathrm{eq}}\left(x_{\mathrm{fr}}\right)$ [34. This gives

$$
\begin{aligned}
\frac{1}{Y_{\chi_{\infty}}}-\frac{1}{Y_{\chi}\left(x_{\mathrm{fr}}\right)} & =\int_{x_{\mathrm{fr}}}^{\infty} \frac{\lambda}{x^{2}} \mathrm{~d} x \\
& =\sqrt{\frac{45}{\pi}} m_{\mathrm{P}} \int_{0}^{T_{\mathrm{fr}}}\left\langle\sigma_{\mathrm{ann}} v\right\rangle g_{*}^{1 / 2} \mathrm{~d} T
\end{aligned}
$$

Since $Y_{\chi_{\infty}}$ subsequently remains invariant, the number density of the neutralinos today (at $T=T_{0}$ ) is obtained by simply multiplying by the present entropy density, $s\left(T_{0}\right)$, which is given by eq.(21) with $g_{s}\left(T_{0}\right)=3.91$ and $T_{0}=2.735 \pm 0.02{ }^{0} \mathrm{~K}$ 40. The present neutralino energy density is thus given by 


$$
\Omega_{\chi} h^{2}=2.775 \times 10^{8} Y_{\chi_{\infty}}\left(\frac{m_{\chi}}{\mathrm{GeV}}\right) .
$$

The observational error in $T_{0}$ corresponds to an uncertainty of $\pm 2 \%$ in $\Omega_{\chi} h^{2}$. The above procedure give an accuracy of better than $5 \%$ when compared with numerical solutions of eq. 25) (e.g. 38]).

Two further remarks are in order. We have assumed above that the neutralino, as the LSP, is absolutely stable by virtue of R-parity invariance. However, most models have been considered as effective theories derived from broken GUTs; in some cases (e.g the minimal flipped SU(5) model) a small amount of R-parity breaking is possible, causing the LSP to decay via superheavy boson exchange. As noted earlier, if such metastable LSPs constitute the dark matter, then constraints from astrophysical data [28] require their lifetime to be at least $10^{6}$ times longer than the age of the universe, hence they may be considered effectively stable.

We have also assumed that the neutralino is much lighter than the charginos and sleptons, so that it cannot annihilate into these particles at the epoch of freeze-out; such 'co-annihilation' effects can greatly reduce the relic abundance [37. In fact such effects are important and must be taken into account for pure Higgsino states ref. [41]. However in the present case the neutralino is either a gaugino or a mixed state over most of parameter space. Also, the presence of the gauge singlet ensures that there is no degeneracy between the neutralino and the squark masses as argued in ref. 41] for the case of the MSSM.

\section{Exploring Parameter Space}

We now take the most general expression for the cross-section and composition of the LSP and analyse the parameter space in the $\left(\lambda_{7}, \lambda_{8}\right),\left(m_{0}, m_{1 / 2}\right),\left(\tan \beta, \tan \beta_{x}\right)$ and $(\tan \beta, A)$ planes. Below the $\mathrm{W}$ mass, the calculation of the cross-section is simplified since the contributing diagrams are only those shown in fig. 1 (where $\mathrm{f}$ denotes a matter fermion). The cross section may therefore be found as a general expression involving left and right external fields and arbitrary internal propagators; summing over all mass eigenstates and interactions yields the required quantity (which is far too cumbersome to be presented here). We have checked that our cross sections for various pure LSP states agree with those found in refs. [14, 38].

We restrict our attention to SUSY breaking parameters less than $500 \mathrm{GeV}$, to avoid excessive fine-tuning in the model. \& As discussed earlier, we look for cosmologically 'interesting' regions in which the value of the relic density is $0.1 \lesssim \Omega_{\chi} h^{2} \lesssim 0.5$, and exclude regions in which $\Omega_{\chi} h^{2} \gtrsim 1$. Using recent experimental data from LEP [43] and CDF [44, we also exclude regions of parameter space where the slepton, sneutrino, chargino, (lightest) Higgs or gluino have masses which have already been excluded (or are less than that of the neutralino), or where the supersymmetric contribution [6, 25] to the (recently revised) $\mathrm{Z}^{0}$ 'invisible width' [45] is excessive. In particular, we impose the following bounds (all at the $90 \%$ confidence level):

\footnotetext{
${ }^{2}$ For a model-independent survey see ref. [42].
} 
$m_{\tilde{l}, \chi^{ \pm}}>45 \mathrm{GeV}, \quad \mathrm{m}_{\mathrm{h}}>43 \mathrm{GeV}, \quad \mathrm{m}_{\tilde{\nu}}>40 \mathrm{GeV}, \quad \mathrm{m}_{\tilde{\mathrm{g}}}>150 \mathrm{GeV}, \quad\left(\mathrm{N}_{\nu}-3\right)<0.11$

We also adopt $m_{\mathrm{t}}=120 \mathrm{GeV}$ as suggested by recent analyses of high precision LEP data [46]. It is neccessary to exclude negative Higgs and pseudo-Higgs masses-squared since otherwise the absolute minimum of the potential would be CP-violating, as is implicit in their mass matrices [11].

\subsection{The $\left(\lambda_{7}, \lambda_{8}\right)$ plane}

Since the sfermion masses are independent of $\lambda_{7}$ and $\lambda_{8}$ to first order (see the appendices), when we look in the $\left(\lambda_{7}, \lambda_{8}\right)$ plane we see the effect on the LSP relic density of the masses of the LSP, Higgs and pseudo-Higgs only. (In the two remaining planes, however, the masses of all the (s)particles in the low energy spectrum have an effect.) For a gauginolike LSP, sfermion exchange is the important contribution to $\left\langle\sigma_{\text {ann }} v\right\rangle$, but Z exchange becomes increasingly important for Higgsino-like and mixed LSPs. Also, for Higgsino-like LSPs, the sfermion exchange diagrams are suppressed by a factor of at least $m_{\mathrm{b}} / m_{\mathrm{Z}}$.

In contrast with previous work, it is not possible here to choose the signs of $A_{7}$ and $A_{8}$, in order to guarantee the positiveness of the Higgs and pseudo-Higgs masses-squared. Instead we choose the signs of the VEVs such that the experimental conditions above are satisfied, and allow $\lambda_{7}$ and $\lambda_{8}$ to be both positive and negative. This translates to the following constraints on the VEVs:

$$
\begin{aligned}
& \operatorname{Sgn}[\bar{v}]=+1 \\
& \operatorname{Sgn}[v]=-\operatorname{Sgn}\left[\lambda_{7} \lambda_{8}\right] \\
& \operatorname{Sgn}[x]=+\operatorname{Sgn}\left[A \lambda_{8}\right] .
\end{aligned}
$$

We find no other choice of parameters which consistently meet all experimental requirements. The first condition is chosen arbitrarily, without loss of generality. Inspection of the neutral terms in the potential shows that under the above conditions, all the masses are invariant under the transformations $\lambda_{7} \rightarrow-\lambda_{7}$ and $\lambda_{8} \rightarrow-\lambda_{8}$. We are therefore free to choose $\lambda_{7}$ and $\lambda_{8}$ to be positive.

In figs. 2a-d we show contours of the LSP mass and the relic density in the $\left(\lambda_{7}-\lambda_{8}\right)$ plane, as well as the experimentally allowed regions. (Note that $\lambda_{7}$ and $\lambda_{8}$ are bounded by their infra-red fixed values of 0.87 and 0.21 respectively). We choose $m_{0}$ and $m_{1 / 2}$ to be comparable and of order the Fermi scale since the changing LSP mass is then the dominant parameter in determining the relic density. For small values of $\lambda_{7}$ and $\lambda_{8}$, the LSP mass is small and the relic abundance large. However these regions are unlikely to satisfy experimental bounds and we find that this is indeed so. At large $\lambda_{7}$ and $\lambda_{8}$, the Higgs and pseudo-Higgs masses increase and therefore do not contribute appreciably to $\left\langle\sigma_{\text {ann }} v\right\rangle$, which is then dominated by sfermion exchange. Also the LSP becomes mostly gaugino, and its mass becomes large and nearly constant. For positive $A$, the relic density 
tends to a plateau (at a relatively low value) in the experimentally allowed region (see fig. 2a). (The 'holes' seen in the figures correspond to poles in the annihilation crosssection where the calculated relic density is neligibly small. As noted earlier, this is an artifact of the approximate series expansion eq. (18); correct thermal averaging of the cross-section at resonances shows that the dips in the relic abundance are not in fact as sharp or as deep [36, 37.)

The behaviour is similar, although somewhat more complex, for negative $A$, but the more mixed LSP states produce a significantly lower relic density in the experimentally allowed region. Higher values of $\Omega_{\chi} h^{2}$ occur along the strips where $\lambda_{7} \sim 0.6$, and where $\lambda_{7} \sim 0.25$, when the LSP mass is still relatively small (see fig. $2 \mathrm{~b}$ ). Because of the relations in eq. (32), the relic density takes longer to reach its plateau at high values of the Yukawa couplings since the LSP eigenstate is dominated by the mixed higgsino/singlet.

In addition, making $|A|, \tan \beta$ or $m_{0}$ large causes the relic density to flatten out more quickly, and tends to increase its value in the flat region (see figs. 2c,d). We find that the experimentally allowed region tends to shrink for higher values of $\tan \beta$ and $|A|$ and therefore conclude that these are experimentally disfavoured. These last two observations will be discussed in more detail below.

For the values of parameters chosen in fig. 2 d (i.e. $A=+1, \tan \beta=4, \tan \beta_{x}=1 / 2$, $m_{\mathrm{t}}=120 \mathrm{GeV}, m_{0}=300 \mathrm{GeV}$ and $\left.m_{1 / 2}=200 \mathrm{GeV}\right), \Omega_{\chi} h^{2}$ flattens out at

$$
\Omega_{\chi} h^{2} \simeq 0.4
$$

as seen in fig. 2e. In the experimentally allowed region, there is very little variation with $\lambda_{8}$, for either positive or negative $A$. In this region the neutralino mass lies in the range $\sim 60-80 \mathrm{GeV}$.

Clearly the large value of $\lambda_{7}\left(=g_{2}\right)$ often chosen in the literature [14] is in fact favoured by experiment; also the relic density in this region is particularly constant. The value of $\lambda_{8} \sim g_{2} / 6$ [14] however seems to be too low, and $\lambda_{8} \sim 0.2$ is a better choice. (In any case the former value was derived using smaller values of the top quark coupling, and we find that in our case the latter value is quite natural for $\lambda_{2 \mathrm{t}}$ of $\mathcal{O}(1)$ at the GUT scale.)

Henceforth, we shall choose $\lambda_{7}=g_{2}$ and $\lambda_{8}=0.2$, to analyse the $\left(m_{0}, m_{1 / 2}\right)$ and $(\tan \beta$, $\tan \beta_{x}$ ) planes, bearing in mind that when the LSP contains a significant proportion of higgsino or gauge singlet, the values obtained for $\Omega_{\chi} h^{2}$ may be slight grey reduced by choosing larger $\lambda_{7}$.

\subsection{The $\left(m_{0}, m_{1 / 2}\right)$ plane}

In figs. 3a-d we exhibit contours of the relic density, together with the experimentally allowed region, and the mass of the LSP (which depends only on the value of $m_{1 / 2}$ ). There are three easily identifiable regions in the $\left(m_{0}, m_{1 / 2}\right)$ plane. The first is the region close to the origin, where the relic density is small. This is because here the sfermion, Higgs and pseudo-Higgs masses are small, causing $\left\langle\sigma_{\text {ann }} v\right\rangle$ to be correspondingly large. The LSP mass increases linearly with $m_{1 / 2}$, until it becomes dominated by the gauge singlet, when it becomes constant. At this point the relic density tends to increase, 
as the sfermion exchange contribution to $\left\langle\sigma_{\text {ann }} v\right\rangle$ (the third diagram of fig. 1) becomes suppressed. This increase becomes more marked for larger $\tan \beta$, and as a result, the region of high $m_{1 / 2}(\gtrsim 300 \mathrm{GeV})$ appears to be excluded for these values. The third region one can identify is where $m_{0}$ is large, the LSP mass is small, and the Higgs and pseudo-Higgs masses are large. This also yields a cosmologically interesting relic density for $m_{0} \gtrsim 200 \mathrm{GeV}$, which is relatively independent of $\tan \beta$ and $A$. This behaviour is similar to that found for the MSSM in ref. [9]. At low $m_{1 / 2}$ the LSP is mostly photino and has a mass of order $\frac{2}{3} \sin ^{2} \theta_{\mathrm{W}} m_{2}+\mathcal{O}\left(m_{2}^{2} / m_{\mathrm{Z}}\right)$, in accord with ref. [14].

We find that high values of $\Omega_{\chi} h^{2}$ are favoured by high values of $m_{0}$ and low values of $m_{1 / 2}$. In particular we note that for $\tan \beta \gtrsim 4$ and negative $A$, there are no allowed regions which are cosmologically interesting. For positive $A$, the no-scale theories $\left(m_{0}=0\right)$ are excluded by LEP as shown in fig. 3a. Note that since we are using the choice of VEVs given by eq. (32), the sign of $A$ may instead be regarded as a choice of the sign of $\lambda_{8}$. For negative $A$ this is not the case, and we find that there exists an upper limit on the allowed $m_{0}$, coming from the requirement that the vacuum not be $\mathrm{CP}$-violating (see fig. $3 \mathrm{~b}$ ). This bound becomes more restrictive if the values of $\tan \beta$ or $|A|$ are increased as seen in figs. $3 \mathrm{c}, \mathrm{d}$.

\subsection{The $\left(\tan \beta, \tan \beta_{x}\right)$ and $(\tan \beta, A)$ planes}

Choosing the above values of $\lambda_{7}$ and $\lambda_{8}$, we examine the relic density in the $\left(\tan \beta, \tan \beta_{x}\right)$ plane (figs. 4a,b) for $A= \pm 1, m_{1 / 2}=200 \mathrm{GeV}$, and $m_{0}=300 \mathrm{GeV}$. The experimental constraints discussed earlier now place the following limits on the allowed values of these parameters,

$$
\begin{aligned}
0.5 \lesssim \quad \tan \beta & \lesssim 5.0 \\
\tan \beta_{x} & \lesssim 2.0,
\end{aligned}
$$

corresponding to the values of $\lambda_{7}$ and $\lambda_{8}$ chosen here. (Higher values of $\tan \beta$ considerably shrink the experimentally allowed regions in fig. 2.) These limits are considerably less restrictive than those suggested in ref. [14], where the specific values chosen were $\lambda_{7}=g_{2}$ and $\lambda_{8}=g_{2} / 6 \simeq 0.108$. In addition we find that, remarkably enough, the parameter space is restricted to those values of $\tan \beta$ and $\tan \beta_{x}$ which yield $\Omega_{\chi} \sim 1$, and where the relic density is relatively uniform, especially for positive $A$. In fig. 5 , we examine the effect of varying $A$ and $\tan \beta$. Now $\Omega_{\chi} h^{2}$ is relatively constant over the whole plane, apart from at $A=0$ where we find a jump in the relic density from $\Omega_{\chi} h^{2} \sim 0.3$ down to $\Omega_{\chi} h^{2} \sim 0.1$ due to the change in the sign of $\bar{v}$ and $v$ dictated by eq. (32). In general we conclude that negative values of $A$ favour higher values of $\Omega_{\chi}$. We can place additional limits on the trilinear coupling,

$$
-3 \lesssim A \lesssim 4
$$

which marginally favour positive values of $A$. 


\section{Conclusions}

Using a set of approximate analytic solutions to the RGEs, we have examined the relic density of the LSP in a class of unified theories in which the electroweak symmetry is broken by a gauge singlet. We have used the most general expressions for all the sparticle masses and for the thermally-averaged cross-section below the $\mathrm{W}$ mass. This is particularly important when the LSP is gaugino-like, in which case the dominant contribution to the cross-section is the sfermion exchange. In addition, we stress the importance of using accurate expressions for the scalar and trilinear coupling terms $A_{7}$ and $A_{8}$, since these are involved in determining the electroweak breaking. The unknown Yukawa couplings $\lambda_{7}$ and $\lambda_{8}$ are experimentally restricted to have relatively high values,

$$
\begin{aligned}
& 0.2 \lesssim \lambda_{7} \lesssim \quad 0.8 \\
& 0.1 \lesssim \lambda_{8}<0.21
\end{aligned}
$$

where the last limit is a unitarity constraint, above which the naïve unification assumption is no longer valid. For the chosen values of $m_{\mathrm{t}}=120 \mathrm{GeV}, \lambda_{7}=g_{2}, \lambda_{8}=0.2$, the values of the VEVs are constrained by eq. (34) and the trilinear coupling $A$ at the GUT scale by eq. (35). Further, positive values of $A$ are marginally favoured by experiment and lead to a more uniform relic abundance in parameter space. We find that cosmologically interesting regions exist with

$$
250 \mathrm{GeV} \lesssim m_{0} \lesssim 500 \mathrm{GeV}
$$

For negative $A$, higher values of $m_{0}$ tend to be excluded by symmetry breaking requirements. The region $m_{1 / 2} \gtrsim 300 \mathrm{GeV}$ is excluded for larger $\tan \beta$, although one should now

take into account new processes involving W final states, and would therefore expect $\Omega_{\chi} h^{2}$ to be smaller than our estimates in this region. As pointed out in ref. [14], for various 'pure' LSP states (no gaugino content) there are cosmologically interesting regions for much higher values of $m_{1 / 2}$, although such large masses (exceeding $1 \mathrm{TeV}$ ) necessitate fine tuning of at least one part in a hundred in the top quark Yukawa coupling, to obtain satisfactory electroweak breaking 42]. (We also confirm the conclusion of ref. [10] that for a higgsino-like LSP (corresponding to large $m_{1 / 2}$ and $m_{0}$ and small $\lambda_{8}$ ) there is no bound on the LSP mass coming from the constraint $\Omega_{\chi} h^{2} \lesssim 1$.) Although computational constraints dictated a tree-level treatment of electroweak symmetry breaking in this work, we note that 1-loop corrections have a relatively minor effect on the relic density [14]. We therefore anticipate that our conclusions will remain unaltered in a more detailed analysis of this model including radiative corrections.

Acknowledgement We would like to thank Graham Ross for useful discussions. SAA was supported by a SERC fellowship. 


\section{Appendix A}

Analytic approximations for all required parameters (at $m_{\mathrm{Z}}$ ) in terms of the Yukawa couplings (at $m_{\mathrm{Z}}$ ) and the supersymmetry-breaking terms $m_{0}, m_{1 / 2}$ and $A$ for the minimally extended, supersymmetric, standard model, with $\alpha_{\mathrm{s}}=0.118$. Note that $m_{\mathrm{U}}^{2}, m_{\mathrm{Q}}^{2}, m_{\mathrm{D}}^{2}, \lambda_{1}$ and $\lambda_{2}$ carry suppressed generation indices. [3

$$
\begin{aligned}
& A_{1}=A m_{0}\left(1-0.78 \lambda_{1}^{2}-0.13 \lambda_{2}^{2}-0.31 \lambda_{7}^{2}+0.03 \lambda_{1}^{2} \lambda_{7}^{2}-0.02 \lambda_{2}^{2} \lambda_{7}^{2}+0.25 \lambda_{7}^{2} \lambda_{8}^{2}\right) \\
& +m_{1 / 2}\left(-3.85+1.69 \lambda_{1}^{2}+0.01 \lambda_{1}^{4}+0.28 \lambda_{2}^{2}+0.11 \lambda_{7}^{2}+0.04 \lambda_{1}^{2} \lambda_{7}^{2}-0.02 \lambda_{2}^{2} \lambda_{7}^{2}+0.25 \lambda_{7}^{2} \lambda_{8}^{2}\right) \\
& A_{2}=A m_{0}\left(1-0.13 \lambda_{1}^{2}-0.77 \lambda_{2}^{2}-0.31 \lambda_{7}^{2}-0.02 \lambda_{1}^{2} \lambda_{7}^{2}+0.03 \lambda_{2}^{2} \lambda_{7}^{2}+0.25 \lambda_{7}^{2} \lambda_{8}^{2}\right) \\
& +m_{1 / 2}\left(-3.89+0.28 \lambda_{1}^{2}+1.70 \lambda_{2}^{2}+0.01 \lambda_{2}^{4}+0.11 \lambda_{7}^{2}-0.02 \lambda_{1}^{2} \lambda_{7}^{2}+0.04 \lambda_{2}^{2} \lambda_{7}^{2}+0.25 \lambda_{7}^{2} \lambda_{8}^{2}\right) \\
& A_{7}=A m_{0}\left(1-0.39 \lambda_{1}^{2}-0.39 \lambda_{2}^{2}-1.23 \lambda_{7}^{2}-0.11 \lambda_{1}^{2} \lambda_{7}^{2}-0.11 \lambda_{2}^{2} \lambda_{7}^{2}-7.46 \lambda_{8}^{2}-0.52 \lambda_{7}^{2} \lambda_{8}^{2}\right) \\
& +m_{1 / 2}\left(-0.58+0.85 \lambda_{1}^{2}+0.85 \lambda_{2}^{2}+0.43 \lambda_{7}^{2}-0.10 \lambda_{1}^{2} \lambda_{7}^{2}-0.10 \lambda_{2}^{2} \lambda_{7}^{2}-0.50 \lambda_{7}^{2} \lambda_{8}^{2}\right) \\
& A_{8}=A m_{0}\left(1-1.84 \lambda_{7}^{2}-0.22 \lambda_{1}^{2} \lambda_{7}^{2}-0.22 \lambda_{2}^{2} \lambda_{7}^{2}-22.4 \lambda_{8}^{2}-3.05 \lambda_{7}^{2} \lambda_{8}^{2}\right) \\
& +m_{1 / 2}\left(0.69 \lambda_{7}^{2}-0.21 \lambda_{1}^{2} \lambda_{7}^{2}-0.21 \lambda_{2}^{2} \lambda_{7}^{2}-3.01 \lambda_{7}^{2} \lambda_{8}^{2}\right) \\
& m_{\mathrm{U}}^{2}=m_{0}^{2}\left(1-0.77 \lambda_{2}^{2}-0.26 A^{2} \lambda_{2}^{2}+0.03 A^{2} \lambda_{1}^{2} \lambda_{2}^{2}+0.20 A^{2} \lambda_{2}^{4}+0.07 \lambda_{2}^{2} \lambda_{7}^{2}+0.13 A^{2} \lambda_{2}^{2} \lambda_{7}^{2}\right) \\
& +A m_{0} m_{1 / 2}\left(1.14 \lambda_{2}^{2}-0.15 \lambda_{1}^{2} \lambda_{2}^{2}-0.86 \lambda_{2}^{4}-0.25 \lambda_{2}^{2} \lambda_{7}^{2}\right) \\
& +m_{1 / 2}^{2}\left(6.27-3.21 \lambda_{2}^{2}+0.15 \lambda_{1}^{2} \lambda_{2}^{2}+0.94 \lambda_{2}^{4}-0.07 \lambda_{2}^{2} \lambda_{7}^{2}\right) \\
& m_{\mathrm{Q}}^{2}=m_{0}^{2}\left(1-0.39 \lambda_{1}^{2}-0.13 A^{2} \lambda_{1}^{2}+0.10 A^{2} \lambda_{1}^{4}-0.39 \lambda_{2}^{2}-0.13 A^{2} \lambda_{2}^{2}+0.03 A^{2} \lambda_{1}^{2} \lambda_{2}^{2}+0.10 A^{2} \lambda_{2}^{4}+\right. \\
& \left.0.03 \lambda_{1}^{2} \lambda_{7}^{2}+0.06 A^{2} \lambda_{1}^{2} \lambda_{7}^{2}+0.04 \lambda_{2}^{2} \lambda_{7}^{2}+0.06 A^{2} \lambda_{2}^{2} \lambda_{7}^{2}\right) \\
& +A m_{0} m_{1 / 2}\left(0.56 \lambda_{1}^{2}-0.44 \lambda_{1}^{4}+0.57 \lambda_{2}^{2}-0.14 \lambda_{1}^{2} \lambda_{2}^{2}-0.43 \lambda_{2}^{4}-0.13 \lambda_{1}^{2} \lambda_{7}^{2}-0.13 \lambda_{2}^{2} \lambda_{7}^{2}\right) \\
& +m_{1 / 2}^{2}\left(6.68-1.59 \lambda_{1}^{2}+0.46 \lambda_{1}^{4}-1.60 \lambda_{2}^{2}+0.16 \lambda_{1}^{2} \lambda_{2}^{2}+0.47 \lambda_{2}^{4}-0.03 \lambda_{1}^{2} \lambda_{7}^{2}-0.03 \lambda_{2}^{2} \lambda_{7}^{2}\right) \\
& m_{\mathrm{D}}^{2}=m_{0}^{2}\left(1-0.78 \lambda_{1}^{2}-0.26 A^{2} \lambda_{1}^{2}+0.20 A^{2} \lambda_{1}^{4}+0.03 A^{2} \lambda_{1}^{2} \lambda_{2}^{2}+0.07 \lambda_{1}^{2} \lambda_{7}^{2}+0.13 A^{2} \lambda_{1}^{2} \lambda_{7}^{2}\right) \\
& +A m_{0} m_{1 / 2}\left(1.13 \lambda_{1}^{2}-0.87 \lambda_{1}^{4}-0.14 \lambda_{1}^{2} \lambda_{2}^{2}-0.25 \lambda_{1}^{2} \lambda_{7}^{2}\right) \\
& +m_{1 / 2}^{2}\left(6.22-3.17 \lambda_{1}^{2}+0.93 \lambda_{1}^{4}+0.16 \lambda_{1}^{2} \lambda_{2}^{2}-0.07 \lambda_{1}^{2} \lambda_{7}^{2}\right) \\
& m_{\mathrm{E}}^{2}=m_{0}^{2}+0.15 m_{1 / 2}^{2} \\
& m_{\mathrm{L}}^{2}=m_{0}^{2}+0.51 m_{1 / 2}^{2} \\
& m_{\mathrm{Y}}=0.41 m_{1 / 2} \\
& m_{2}=0.79 m_{1 / 2} \\
& m_{3}=2.77 m_{1 / 2}
\end{aligned}
$$

\footnotetext{
${ }^{3}$ The equivalent expressions for the MSSM may be obtained by setting $\lambda_{7}=\lambda_{8}=0$, replacing $A_{7}$ by $B_{\mu}$ and the first term in $B_{\mu}$ by $B m_{0}$, where $B$ is the quadratic scalar Higgs coupling at the GUT scale.
} 


\section{Appendix B}

As in Appendix A, with $\alpha_{\mathrm{s}}=0.125$.

$$
\begin{aligned}
& A_{1}=A m_{0}\left(1-0.76 \lambda_{1}^{2}-0.12 \lambda_{2}^{2}-0.31 \lambda_{7}^{2}+0.04 \lambda_{1}^{2} \lambda_{7}^{2}-0.02 \lambda_{2}^{2} \lambda_{7}^{2}+0.26 \lambda_{7}^{2} \lambda_{8}^{2}\right) \\
& +m_{1 / 2}\left(-4.09+1.75 \lambda_{1}^{2}+0.01 \lambda_{1}^{4}+0.29 \lambda_{2}^{2}+0.11 \lambda_{7}^{2}+0.04 \lambda_{1}^{2} \lambda_{7}^{2}-0.02 \lambda_{2}^{2} \lambda_{7}^{2}+0.26 \lambda_{7}^{2} \lambda_{8}^{2}\right) \\
& A_{2}=A m_{0}\left(1-0.13 \lambda_{1}^{2}-0.75 \lambda_{2}^{2}-0.31 \lambda_{7}^{2}-0.02 \lambda_{1}^{2} \lambda_{7}^{2}+0.04 \lambda_{2}^{2} \lambda_{7}^{2}+0.26 \lambda_{7}^{2} \lambda_{8}^{2}\right) \\
& +m_{1 / 2}\left(-4.13+0.29 \lambda_{1}^{2}+1.75 \lambda_{2}^{2}+0.01 \lambda_{2}^{4}+0.11 \lambda_{7}^{2}-0.02 \lambda_{1}^{2} \lambda_{7}^{2}+0.04 \lambda_{2}^{2} \lambda_{7}^{2}+0.26 \lambda_{7}^{2} \lambda_{8}^{2}\right) \\
& A_{7}=A m_{0}\left(1-0.38 \lambda_{1}^{2}-0.37 \lambda_{2}^{2}-1.23 \lambda_{7}^{2}-0.11 \lambda_{1}^{2} \lambda_{7}^{2}-0.11 \lambda_{2}^{2} \lambda_{7}^{2}-7.51 \lambda_{8}^{2}-0.48 \lambda_{7}^{2} \lambda_{8}^{2}+0.32 \lambda_{8}^{4}\right) \\
& +m_{1 / 2}\left(-0.59+0.87 \lambda_{1}^{2}+0.88 \lambda_{2}^{2}+0.44 \lambda_{7}^{2}-0.11 \lambda_{1}^{2} \lambda_{7}^{2}-0.11 \lambda_{2}^{2} \lambda_{7}^{2}-0.52 \lambda_{7}^{2} \lambda_{8}^{2}\right) \\
& A_{8}=A m_{0}\left(1-1.85 \lambda_{7}^{2}-0.22 \lambda_{1}^{2} \lambda_{7}^{2}-0.22 \lambda_{2}^{2} \lambda_{7}^{2}-22.5 \lambda_{8}^{2}-3.0 \lambda_{7}^{2} \lambda_{8}^{2}+0.95 \lambda_{8}^{4}\right) \\
& +m_{1 / 2}\left(0.66 \lambda_{7}^{2}-0.22 \lambda_{1}^{2} \lambda_{7}^{2}-0.22 \lambda_{2}^{2} \lambda_{7}^{2}-3.10 \lambda_{7}^{2} \lambda_{8}^{2}\right) \\
& m_{\mathrm{U}}^{2}=m_{0}^{2}\left(1-0.75 \lambda_{2}^{2}-0.25 A^{2} \lambda_{2}^{2}+0.03 A^{2} \lambda_{1}^{2} \lambda_{2}^{2}+0.19 A^{2} \lambda_{2}^{4}+0.07 \lambda_{2}^{2} \lambda_{7}^{2}+0.12 A^{2} \lambda_{2}^{2} \lambda_{7}^{2}\right) \\
& +A m_{0} m_{1 / 2}\left(1.17 \lambda_{2}^{2}-0.15 \lambda_{1}^{2} \lambda_{2}^{2}-0.87 \lambda_{2}^{4}-0.26 \lambda_{2}^{2} \lambda_{7}^{2}\right) \\
& +m_{1 / 2}^{2}\left(6.90-3.44 \lambda_{2}^{2}+0.17 \lambda_{1}^{2} \lambda_{2}^{2}+1.01 \lambda_{2}^{4}-0.07 \lambda_{2}^{2} \lambda_{7}^{2}\right) \\
& m_{\mathrm{Q}}^{2}=m_{0}^{2}\left(1-0.38 \lambda_{1}^{2}-0.13 A^{2} \lambda_{1}^{2}+0.10 A^{2} \lambda_{1}^{4}-0.37 \lambda_{2}^{2}-0.12 A^{2} \lambda_{2}^{2}+0.03 A^{2} \lambda_{1}^{2} \lambda_{2}^{2}+0.10 A^{2} \lambda_{2}^{4}+\right. \\
& \left.0.04 \lambda_{1}^{2} \lambda_{7}^{2}+0.06 A^{2} \lambda_{1}^{2} \lambda_{7}^{2}+0.04 \lambda_{2}^{2} \lambda_{7}^{2}+0.06 A^{2} \lambda_{2}^{2} \lambda_{7}^{2}\right) \\
& +A m_{0} m_{1 / 2}\left(0.58 \lambda_{1}^{2}-0.44 \lambda_{1}^{4}+0.58 \lambda_{2}^{2}-0.15 \lambda_{1}^{2} \lambda_{2}^{2}-0.43 \lambda_{2}^{4}-0.13 \lambda_{1}^{2} \lambda_{7}^{2}-0.13 \lambda_{2}^{2} \lambda_{7}^{2}\right) \\
& +m_{1 / 2}^{2}\left(7.30-1.71 \lambda_{1}^{2}+0.50 \lambda_{1}^{4}-1.72 \lambda_{2}^{2}+0.17 \lambda_{1}^{2} \lambda_{2}^{2}+0.50 \lambda_{2}^{4}-0.03 \lambda_{1}^{2} \lambda_{7}^{2}-0.03 \lambda_{2}^{2} \lambda_{7}^{2}\right) \\
& m_{\mathrm{D}}^{2}=m_{0}^{2}\left(1-0.76 \lambda_{1}^{2}-0.25 A^{2} \lambda_{1}^{2}+0.19 A^{2} \lambda_{1}^{4}+0.03 A^{2} \lambda_{1}^{2} \lambda_{2}^{2}+0.07 \lambda_{1}^{2} \lambda_{7}^{2}+0.13 A^{2} \lambda_{1}^{2} \lambda_{7}^{2}\right) \\
& +A m_{0} m_{1 / 2}\left(1.16 \lambda_{1}^{2}-0.88 \lambda_{1}^{4}-0.14 \lambda_{1}^{2} \lambda_{2}^{2}-0.26 \lambda_{1}^{2} \lambda_{7}^{2}\right) \\
& +m_{1 / 2}^{2}\left(6.84-3.41 \lambda_{1}^{2}+1.0 \lambda_{1}^{4}+0.17 \lambda_{1}^{2} \lambda_{2}^{2}-0.07 \lambda_{1}^{2} \lambda_{7}^{2}\right) \\
& m_{\mathrm{E}}^{2}=m_{0}^{2}+0.15 m_{1 / 2}^{2} \\
& m_{\mathrm{L}}^{2}=m_{0}^{2}+0.52 m_{1 / 2}^{2} \\
& m_{\mathrm{Y}}=0.40 m_{1 / 2} \\
& m_{2}=0.78 m_{1 / 2} \\
& m_{3}=2.89 m_{1 / 2}
\end{aligned}
$$




\section{Appendix $\mathrm{C}$}

As in Appendix A, with $\alpha_{\mathrm{s}}=0.113$.

$$
\begin{aligned}
& A_{1}=A m_{0}\left(1-0.80 \lambda_{1}^{2}-0.13 \lambda_{2}^{2}-0.30 \lambda_{7}^{2}+0.03 \lambda_{1}^{2} \lambda_{7}^{2}-0.02 \lambda_{2}^{2} \lambda_{7}^{2}+0.24 \lambda_{7}^{2} \lambda_{8}^{2}\right) \\
& +m_{1 / 2}\left(-3.65+1.64 \lambda_{1}^{2}+0.01 \lambda_{1}^{4}+0.28 \lambda_{2}^{2}+0.10 \lambda_{7}^{2}+0.04 \lambda_{1}^{2} \lambda_{7}^{2}-0.02 \lambda_{2}^{2} \lambda_{7}^{2}+0.24 \lambda_{7}^{2} \lambda_{8}^{2}\right) \\
& A_{2}=A m_{0}\left(1-0.13 \lambda_{1}^{2}-0.79 \lambda_{2}^{2}-0.30 \lambda_{7}^{2}-0.02 \lambda_{1}^{2} \lambda_{7}^{2}+0.03 \lambda_{2}^{2} \lambda_{7}^{2}+0.24 \lambda_{7}^{2} \lambda_{8}^{2}\right) \\
& +m_{1 / 2}\left(-3.69+0.27 \lambda_{1}^{2}+1.65 \lambda_{2}^{2}+0.01 \lambda_{2}^{4}+0.10 \lambda_{7}^{2}-0.02 \lambda_{1}^{2} \lambda_{7}^{2}+0.04 \lambda_{2}^{2} \lambda_{7}^{2}+0.24 \lambda_{7}^{2} \lambda_{8}^{2}\right) \\
& A_{7}=A m_{0}\left(1-0.40 \lambda_{1}^{2}-0.39 \lambda_{2}^{2}-1.21 \lambda_{7}^{2}-0.11 \lambda_{1}^{2} \lambda_{7}^{2}-0.11 \lambda_{2}^{2} \lambda_{7}^{2}-7.33 \lambda_{8}^{2}-0.47 \lambda_{7}^{2} \lambda_{8}^{2}+0.15 \lambda_{8}^{4}\right) \\
& +m_{1 / 2}\left(-0.57+0.82 \lambda_{1}^{2}+0.83 \lambda_{2}^{2}+0.42 \lambda_{7}^{2}-0.10 \lambda_{1}^{2} \lambda_{7}^{2}-0.10 \lambda_{2}^{2} \lambda_{7}^{2}-0.49 \lambda_{7}^{2} \lambda_{8}^{2}\right) \\
& A_{8}=A m_{0}\left(1-1.82 \lambda_{7}^{2}-0.21 \lambda_{1}^{2} \lambda_{7}^{2}-0.21 \lambda_{2}^{2} \lambda_{7}^{2}-22.0 \lambda_{8}^{2}-2.84 \lambda_{7}^{2} \lambda_{8}^{2}+0.46 \lambda_{8}^{4}\right) \\
& +m_{1 / 2}\left(0.62 \lambda_{7}^{2}-0.21 \lambda_{1}^{2} \lambda_{7}^{2}-0.21 \lambda_{2}^{2} \lambda_{7}^{2}-2.87 \lambda_{7}^{2} \lambda_{8}^{2}\right) \\
& m_{\mathrm{U}}^{2}=m_{0}^{2}\left(1-0.79 \lambda_{2}^{2}-0.26 A^{2} \lambda_{2}^{2}+0.04 A^{2} \lambda_{1}^{2} \lambda_{2}^{2}+0.20 A^{2} \lambda_{2}^{4}+0.07 \lambda_{2}^{2} \lambda_{7}^{2}+0.13 A^{2} \lambda_{2}^{2} \lambda_{7}^{2}\right) \\
& +A m_{0} m_{1 / 2}\left(1.10 \lambda_{2}^{2}-0.14 \lambda_{1}^{2} \lambda_{2}^{2}-0.86 \lambda_{2}^{4}-0.24 \lambda_{2}^{2} \lambda_{7}^{2}\right) \\
& +m_{1 / 2}^{2}\left(5.74-2.99 \lambda_{2}^{2}+0.15 \lambda_{1}^{2} \lambda_{2}^{2}+0.89 \lambda_{2}^{4}-0.06 \lambda_{2}^{2} \lambda_{7}^{2}\right) \\
& m_{\mathrm{Q}}^{2}=m_{0}^{2}\left(1-0.40 \lambda_{1}^{2}-0.13 A^{2} \lambda_{1}^{2}+0.11 A^{2} \lambda_{1}^{4}-0.39 \lambda_{2}^{2}-0.13 A^{2} \lambda_{2}^{2}+0.03 A^{2} \lambda_{1}^{2} \lambda_{2}^{2}+0.10 A^{2} \lambda_{2}^{4}+\right. \\
& \left.0.03 \lambda_{1}^{2} \lambda_{7}^{2}+0.06 A^{2} \lambda_{1}^{2} \lambda_{7}^{2}+0.03 \lambda_{2}^{2} \lambda_{7}^{2}+0.06 A^{2} \lambda_{2}^{2} \lambda_{7}^{2}\right) \\
& +A m_{0} m_{1 / 2}\left(0.55 \lambda_{1}^{2}-0.43 \lambda_{1}^{4}+0.55 \lambda_{2}^{2}-0.14 \lambda_{1}^{2} \lambda_{2}^{2}-0.43 \lambda_{2}^{4}-0.12 \lambda_{1}^{2} \lambda_{7}^{2}-0.12 \lambda_{2}^{2} \lambda_{7}^{2}\right) \\
& +m_{1 / 2}^{2}\left(6.15-1.48 \lambda_{1}^{2}+0.44 \lambda_{1}^{4}-1.50 \lambda_{2}^{2}+0.15 \lambda_{1}^{2} \lambda_{2}^{2}+0.44 \lambda_{2}^{4}-0.03 \lambda_{1}^{2} \lambda_{7}^{2}-0.03 \lambda_{2}^{2} \lambda_{7}^{2}\right) \\
& m_{\mathrm{D}}^{2}=m_{0}^{2}\left(1-0.80 \lambda_{1}^{2}-0.27 A^{2} \lambda_{1}^{2}+0.21 A^{2} \lambda_{1}^{4}+0.03 A^{2} \lambda_{1}^{2} \lambda_{2}^{2}+0.07 \lambda_{1}^{2} \lambda_{7}^{2}+0.13 A^{2} \lambda_{1}^{2} \lambda_{7}^{2}\right) \\
& +A m_{0} m_{1 / 2}\left(1.09 \lambda_{1}^{2}-0.86 \lambda_{1}^{4}-0.14 \lambda_{1}^{2} \lambda_{2}^{2}-0.24 \lambda_{1}^{2} \lambda_{7}^{2}\right) \\
& +m_{1 / 2}^{2}\left(5.70-2.97 \lambda_{1}^{2}+0.88 \lambda_{1}^{4}+0.15 \lambda_{1}^{2} \lambda_{2}^{2}-0.06 \lambda_{1}^{2} \lambda_{7}^{2}\right) \\
& m_{\mathrm{E}}^{2}=m_{0}^{2}+0.15 m_{1 / 2}^{2} \\
& m_{\mathrm{L}}^{2}=m_{0}^{2}+0.50 m_{1 / 2}^{2} \\
& m_{\mathrm{Y}}=0.42 m_{1 / 2} \\
& m_{2}=0.79 m_{1 / 2} \\
& m_{3}=2.67 m_{1 / 2}
\end{aligned}
$$




\section{References}

[1] J. Ellis, Phil. Trans. R. Soc. Lond. A336 (1991) 247, Phys. Scr. T36 (1991) 142

[2] H. Goldberg, Phys. Rev. Lett. 50 (1983) 1419;

L.M. Krauss, Nucl. Phys. B227 (1983) 556;

J. Ellis, J.S. Hagelin, D.V. Nanopoulos, K.A. Olive and M. Srednicki, Nucl. Phys. B238 (1984) 453

K. Griest, Phys. Rev. D38 (1988) 2357

[3] K.A. Olive and M.Srednicki, Phys. Lett. 230B (1989) 78;

K.A. Olive and M.Srednicki, Nucl. Phys. B355 (1991) 208;

J.M. McDonald, K.A. Olive and M. Srednicki, Phys. Lett. B283 (1992) 80

[4] K. Griest, M. Kamionkowski and M.S. Turner, Phys. Rev. D41 (1990) 3565

[5] R. Barbieri, M. Frigeni and G. Giudice, Nucl. Phys. B313 (1989) 725;

J. Ellis, L. Roszkowski and Z. Lalak, Phys. Lett. 245B (1990) 545

[6] L. Krauss, Phys. Rev. Lett. 64 (1990) 999;

J. Ellis, D.V. Nanopoulos, D.N. Schramm and L. Roszkowski, Phys. Lett. B245 (1990) 251

[7] L. Roszkowski, Phys. Lett. 262 (1991) 59, Proc. Joint Lepton-Photon Symp. \& Europhys. Conf. on High Energy Phys., Geneva, eds. S. Hegarty et al (World-Scientific, 1992) Vol.1, p.498

[8] J.L. Lopez, K. Yuan and D.V. Nanopoulos, Phys. Lett. 267B (1991) 219;

J.L. Lopez, D.V. Nanopoulos and K. Yuan, Nucl. Phys. B370 (1992) 445;

S. Kelley, J.L. Lopez, D.V. Nanopoulos, H. Pois and K. Yuan, Texas preprint CTPTAMU-56/92 (1992)

[9] J. Ellis and L. Roszkowski, Phys. Lett. 283B (1992) 252

[10] M. Drees and M.M. Nojiri, DESY preprint 92-101/SLAC preprint PUB-5860 (1992)

[11] J. Ellis, J.F. Gunion, H.E. Haber, L. Roszkowski and F. Zwirner, Phys. Rev. D39 (1989) 844

[12] B.R. Greene and P.J. Miron, Phys. Lett. 168B (1986) 226

[13] R. Flores, K.A. Olive and and D. Thomas, Phys. Lett. 245B (1990) 509

[14] K.A. Olive and D. Thomas, Nucl. Phys. B355 (1991) 192

[15] E.W. Kolb and M.S. Turner, The Early Universe (Addison-Wesley, 1990)

[16] J. Binney and S. Tremaine, Galactic Dynamics (Princeton, 1988) 
[17] N. Kaiser, Proc. Texas/ESO-CERN Conf., Brighton, ed. J. Barrow (1992) in press; O. Lahav, in After the First Three Minutes, eds. S.S. Holt et al (AIP, 1991) p.421

[18] E.L. Wright et al, Astrophys. J. 396 (1992) L13;

N. Gouda and N.Sugiyama, Astrophys. J. 395 (1992) L59;

G. Efstathiou, J.R. Bond and S.D.M. White, Mon. Not. R. Astr. Soc. 258 (1992) 1p

[19] J. Ellis, G. Ridolfi and F. Zwirner, Phys. Lett. 262B (1991) 477

[20] J.-P Derendinger and C.A Savoy, Nucl. Phys. B237 (1984) 307

[21] S.M. Barr, Phys. Lett. 112B (1982) 219;

I. Antoniadis, J. Ellis, J.S. Hagelin and D.V. Nanopoulos, Phys. Lett. 194B (1987) 231;

B.A. Campbell, J. Ellis, J.S. Hagelin, D.V. Nanopoulos and R. Ticciati, Phys. Lett. 198B (1987) 200

[22] I. Antoniadis, J. Ellis, J.S. Hagelin and D.V. Nanopoulos, Phys. Lett. 208B (1988) 209 ;

J.L. Lopez and D.V. Nanopoulos, Nucl. Phys. B338 (1990) 73;

J.L. Lopez and D.V. Nanopoulos, Phys. Lett. 251B (1990) 73;

J.L. Lopez and D.V. Nanopoulos, Phys. Lett. 268B (1991) 359

[23] J. Ellis, J.S. Hagelin, S. Kelley and D.V. Nanopoulos, Nucl. Phys. B311 (1988/89) 1

[24] F. Gabbiani and A. Masiero, Phys. Lett. 209B (1988) 289;

S.M. Barr, Phys. Rev. D40 (1989) 2457;

A.E. Faraggi, J.L. Lopez, D.V. Nanopoulos, Phys. Lett. 221B (1989) 337;

S.A. Abel, W.N. Cottingham and I.B. Whittingham, Phys. Lett. 236B (1990) 305;

J. Rizos and K. Tamvakis, Phys. Lett. 251B (1990) 369;

J. Ellis, J.L. Lopez and D.V. Nanopoulos, Phys. Lett. 252B (1990) 53;

S. Kelley, J.L. Lopez and D.V. Nanopoulos, Texas preprint CPT-TAMU-90/90 (1990)

[25] H. Komatsu, Phys. Lett. 177B (1986) 201;

S.A. Abel, W.N. Cottingham and I.B. Whittingham, Phys. Lett. 244B (1990) 327

[26] J. Ellis, J.S. Hagelin, S. Kelley, D.V. Nanopoulos and K.A. Olive, Phys. Lett. 209B (1988) 283;

M. J.McDonald, Phys. Lett. 225B (1989) 133

[27] J. Ellis, J.L. Lopez, and D.V. Nanopoulos, Phys. Lett. 247B (1990) 257

[28] J. Ellis, G.B. Gelmini and S. Sarkar, Nucl. Phys. B373 (1992) 399;

P. Gondolo, G.B. Gelmini and S. Sarkar, UCLA preprint 91/TEP/31 (1991);

S. Sarkar, Nucl. Phys. B (Proc. Suppl.) 28A (1992) 405

[29] S.A. Abel, Ph.D. theis, University of Bristol (1990) unpublished 
[30] A.B. Lahanas and D.V. Nanopoulos, Phys. Rep. 145 (1987) 1

[31] H. Burkhardt and J. Steinberger, Ann. Rev. Nucl. Part. Sci. 41 (1991) 55;

P. Langacker, M. Luo and A. K.Mann, Rev. Mod. Phys. 64 (1992) 87

[32] S. Bethke and S. Catani, CERN preprint TH.6484/92;

S. Bethke, Proc. XXVI Intern. Conf. on High Energy Phys., Dallas (1992) in press

[33] L.E. Ibáñez, C. López and C. Muñoz, Nucl. Phys. B256 (1985) 218

[34] B.W. Lee and S. Weinberg, Phys. Rev.Lett. 39 (1977) 165;

M.I. Vysotskii, A.D. Dolgov and Ya B. Zeldovich, JETP Lett. 26 (1977) 188

[35] J. Bernstein, L.S. Brown and G. Feinberg, Phys. Rev. D32 (1985) 3261;

J. Bernstein, Kinetic Theory in the Expanding Universe, (Cambridge, 1988)

[36] P. Gondolo and G. Gelmini, Nucl. Phys. B360 (1991) 145

[37] K. Griest and D. Seckel, Phys. Rev. D43 (1991) 3191

[38] M. Srednicki, R. Watkins and K.A. Olive, Nucl. Phys. B310 (1988) 693

[39] S. Dimopoulos, R. Esmailzadeh, L.J. Hall and G. Starkman, Phys. Lett. B247 (1990) 601

[40] J.C. Mather et al, Astrophys. J. Lett. 354 (1990) L37;

H.P. Gush, M. Halpern and E.H. Wishnow, Phys. Rev. Lett. 65(1990)537;

R.B. Partridge, in Cosmology and Large-Scale Structure in the Universe, ed. R.R. de Carvalho (ASP, 1992) p.97

[41] S. Mizuta and M. Yamaguchi, Tohoku preprint TU-409 (1992)

[42] G.G. Ross and R.G. Roberts, RAL preprint 92-005 (1992)

[43] M. Davier, Proc. Joint Lepton-Photon Symp. \& Europhys. Conf. on High Energy Phys., Geneva, eds. S. Hegarty et al (World-Scientific, 1991) Vol.2, p.151

[44] A. Laasanen, Proc. Particles and Fields '91, Vancouver (APS, 1992) in press

[45] L. Rolandi, Proc. XXVI Intern. Conf. on High Energy Phys., Dallas (1992) in press

[46] J. Ellis, G. Fogli and E. Lisi, Phys. Lett. B274 (1992) 456;

F. del Aguila, M. Martínez and M. Quirós, Nucl. Phys. B381 (1992) 451 


\section{Figures}

Figure 1 Diagrams contributing to the thermally-averaged cross-section.

Figure 2a Contour plots of $\Omega_{\chi} h^{2}$ and the LSP mass (in GeV) in the $\lambda_{7}-\lambda_{8}$ plane, for $A=+1, \tan \beta=2, \tan \beta_{x}=1 / 2, m_{\mathrm{t}}=120 \mathrm{GeV}, m_{0}=300 \mathrm{GeV}$ and $m_{1 / 2}=$ $200 \mathrm{GeV}$. The cosmologically interesting region $0.1<\Omega_{\chi} h^{2}<0.5$ is shaded light grey while the forbidden region $\Omega_{\chi} h^{2}>1$ is shaded black; the dark grey regions correspond to intermediate values of $\Omega_{\chi} h^{2}$. The bold hatched line encloses the experimentally allowed region. The arrows on the axes indicate the infra-red fixed points.

Figure 2b As in fig. 2a, but with $A=-1$.

Figure 2c As in fig. 2a, but with $A=-3$.

Figure 2d As in fig. 2a, but with $\tan \beta=4$.

Figure 2e $\Omega_{\chi} h^{2}$ versus $\lambda_{7}$ with $\lambda_{8}=0.2$; the other parameters are as in fig. $2 \mathrm{~d}(A=+1$, $\tan \beta=4, \tan \beta_{x}=1 / 2, m_{\mathrm{t}}=120 \mathrm{GeV}, m_{0}=300 \mathrm{GeV}$ and $\left.m_{1 / 2}=200 \mathrm{GeV}\right)$. The experimentally allowed region is demarcated by the vertical hatched lines.

Figure 3a Contour plots of $\Omega_{\chi} h^{2}$ and the LSP mass (in GeV) in the $m_{0}-m_{1 / 2}$ plane, for $A=+1, \tan \beta=2, \tan \beta_{x}=1 / 2, \lambda_{7}=g_{2}, \lambda_{8}=0.2$ and $m_{\mathrm{t}}=120 \mathrm{GeV}$. The cosmologically interesting region $0.1<\Omega_{\chi} h^{2}<0.5$ is shaded light grey while the forbidden region $\Omega_{\chi} h^{2}>1$ is shaded black; the dark grey regions correspond to intermediate values of $\Omega_{\chi} h^{2}$. The bold hatched line encloses the experimentally allowed region.

Figure 3b As in fig. 3a, but with $A=-1$.

Figure 3c As in fig. 3a, but with $A=-3$.

Figure 3d As in fig. 3b, but with $\tan \beta=4$.

Figure 3e $\Omega_{\chi} h^{2}$ versus $m_{0}$ with $m_{1 / 2}=200 \mathrm{GeV}$; the other parameters are as in fig. 3b $\left(A=-1, \tan \beta=2, \tan \beta_{x}=1 / 2, \lambda_{7}=g_{2}, \lambda_{8}=0.2\right.$ and $\left.m_{\mathrm{t}}=120 \mathrm{GeV}\right)$. The experimentally allowed region is demarcated by the vertical hatched lines.

Figure 4a Contour plots of $\Omega_{\chi} h^{2}$ and the LSP mass (in GeV) in the $\tan \beta-\tan \beta_{x}$ plane, for $A=+1, \lambda_{7}=g_{2}, \lambda_{8}=0.2, m_{\mathrm{t}}=120 \mathrm{GeV}, m_{0}=300 \mathrm{GeV}$ and $m_{1 / 2}=200 \mathrm{GeV}$. The cosmologically interesting region $0.1<\Omega_{\chi} h^{2}<0.5$ is shaded light grey while the forbidden region $\Omega_{\chi} h^{2}>1$ is shaded black; the dark grey regions correspond to intermediate values of $\Omega_{\chi} h^{2}$. The bold hatched line encloses the experimentally allowed region.

Figure $4 \mathrm{~b}$ As in fig. 4a, but with $A=-1$. 
Figure 5 Contour plots of $\Omega_{\chi} h^{2}$ and the LSP mass (in $\mathrm{GeV}$ ) in the $\tan \beta-A$ plane, for $\tan \beta_{x}=1 / 2, \lambda_{7}=g_{2}, \lambda_{8}=0.2, m_{\mathrm{t}}=120 \mathrm{GeV}, m_{0}=300 \mathrm{GeV}$ and $m_{1 / 2}=200$ $\mathrm{GeV}$. The cosmologically interesting region $0.1<\Omega_{\chi} h^{2}<0.5$ is shaded light grey while the forbidden region $\Omega_{\chi} h^{2}>1$ is shaded black; the dark regions correspond to intermediate values of $\Omega_{\chi} h^{2}$. The bold hatched line encloses the experimentally allowed region. 